This is the accepted manuscript made available via CHORUS. The article has been published as:

\title{
Direct Measurement of Pyroelectric and Electrocaloric Effects in Thin Films
}

Shishir Pandya, Joshua D. Wilbur, Bikram Bhatia, Anoop R. Damodaran, Christian Monachon, Arvind Dasgupta, William P. King, Chris Dames, and Lane W. Martin

Phys. Rev. Applied 7, 034025 - Published 28 March 2017

DOI: 10.1103/PhysRevApplied.7.034025 


\title{
Direct measurement of pyroelectric and electrocaloric effects in thin films
}

\author{
Shishir Pandya, ${ }^{1}$ Joshua D. Wilbur, ${ }^{2}$ Bikram Bhatia ${ }^{3}$ Anoop R. Damodaran, ${ }^{1}$ Christian \\ Monachon, ${ }^{2}$ Arvind Dasgupta, ${ }^{1}$ William P. King, ${ }^{4}$ Chris Dames, ${ }^{2,5}$ Lane W. Martin ${ }^{1,5},{ }^{*}$ \\ ${ }^{1}$ Materials Science and Engineering, University of California, Berkeley, Berkeley, CA 94720, USA \\ ${ }^{2}$ Mechanical Engineering, University of California, Berkeley, Berkeley, CA 94720, USA \\ ${ }^{3}$ Mechanical Engineering, Massachusetts Institute of Technology, Cambridge, MA 02139, USA \\ ${ }^{4}$ Mechanical Science and Engineering, University of Illinois, \\ Urbana-Champaign, Urbana, IL 61801, USA and

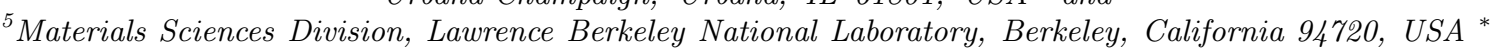

(Dated: February 21, 2017)

\begin{abstract}
Understanding of polarization-heat interactions in pyroelectric and electrocaloric thin-film materials requires that the electrothermal response is reliably characterized. While most work, particularly in electrocalorics, has relied on indirect measurement protocols, here we report a direct technique for measuring both pyroelectric and electrocaloric effects in epitaxial ferroelectric thin films. We demonstrate an electrothermal test platform where localized high-frequency $(\sim 1 \mathrm{kHz})$ periodic heating and highly-sensitive thin-film resistance thermometry allow direct measurement of pyrocurrents $(<10 \mathrm{pA})$ and electrocaloric temperature changes $(<2 \mathrm{mK})$ using the "2-omega" and an adapted "3-omega" technique, respectively. Frequency-domain, phase-sensitive detection permits extraction of pyrocurrent from the total current, which is often convoluted by thermally-stimulated currents. The wide frequency range measurements employed in this study further show the effect of secondary contributions to pyroelectricity due to the mechanical constraints of the substrate. Similarly, measurement of the electrocaloric effect on the same device in the frequency-domain $(\sim 100 \mathrm{kHz})$ allows decoupling of Joule heating from the electrocaloric effect. Using one-dimensional, analytical heattransport models, the transient temperature profile of the heterostructure is characterized to extract pyroelectric and electrocaloric coefficients.
\end{abstract}

\section{INTRODUCTION}

Ferroelectrics possess a spontaneous polarization that is strongly temperature- and electric field-dependent. The variation of polarization with changing temperature results in the pyroelectric effect (PEE), parametrized by the pyroelectric coefficient, $\pi=\left(\frac{\partial P}{\partial T}\right)_{E}$. [1] Application of an electric field can also drive changes in the polarization and the electrocaloric effect (ECE) where application or withdrawal of electric field results in an isothermal entropy $\left(\frac{\partial S}{\partial E}\right)_{T}$ or adiabatic temperature change. [2] Such effects have been widely utilized in a range of applications; pyroelectrics for infrared imaging, radiometry, electron emission, [3, 4] and for the recovery of electrical energy from waste heat [5, [6]; and electrocalorics for environmentally-friendly, solid-state cooling technologies (i.e., low-power, gaseous refrigerant-free, etc.). [7, 8]

The pyroelectric coefficient $\pi$ is typically characterized by measuring the pyroelectric current $\left(i_{P}=A \pi \frac{d T}{d t}\right.$, where $A$ is the area of the capacitor and $\frac{d T}{d t}$ the temperature ramp rate) generated in response to a known temperature transient. Most techniques used to measure pyroelectric properties were developed to probe bulk ceramics or single crystals, including laserinduced heating [9] and continuous ramp-rate heating studies. 10] These techniques are adequate to estimate $\pi$ for large samples (or large area capacitors), but lack the precision, as a consequence of poor tempera-

\footnotetext{
*lwmartin@berkeley.edu
}

ture accuracy, non-uniform heating, and contributions from thermally-stimulated currents due to the release of trapped charges [1, 12], to measure small volume samples (and small area capacitors).[13] For all materials, but particularly for thin-film samples, measurement methods that can separate out deleterious or spurious signals are the key to accurately measuring the true pyroelectric nature. In turn, the work on thin films has turned to phase-sensitive pyroelectric measurement techniques, [14, 15] including those based on microfabricated resistive heater-based measurements [16] and modulated laser-based approaches 17 to more accurately assess the pyroelectric response of these small volume samples.

The ECE, on the other hand, is parametrized by the isothermal entropy change $\left(\Delta S_{i s o}=-\int_{E_{1}}^{E_{2}} \Sigma d E\right)$ where $\Sigma=\left(\frac{\partial S}{\partial E}\right)_{T}$ is the electrocaloric coefficient. In the case of bulk materials, the traditional approach to measuring this quantity is to measure either the change in temperature using thermometry [18] or the heat flux using calorimetry. [19, 20] Similar to the PEE, the last few decades have seen growing interest in investigating the ECE in thin films and, in turn, a need for more advanced measurement approaches has developed. Driven by advances in thin-film epitaxy, researchers can now synthesize ferroelectric thin films and superlattices with atomic-level control over composition and epitaxial strain. 21, 22] It is thought that such thin-film versions of ferroelectrics can undergo significantly larger (orderof-magnitude) temperature changes as they can be driven considerably harder than their bulk counterparts owing 


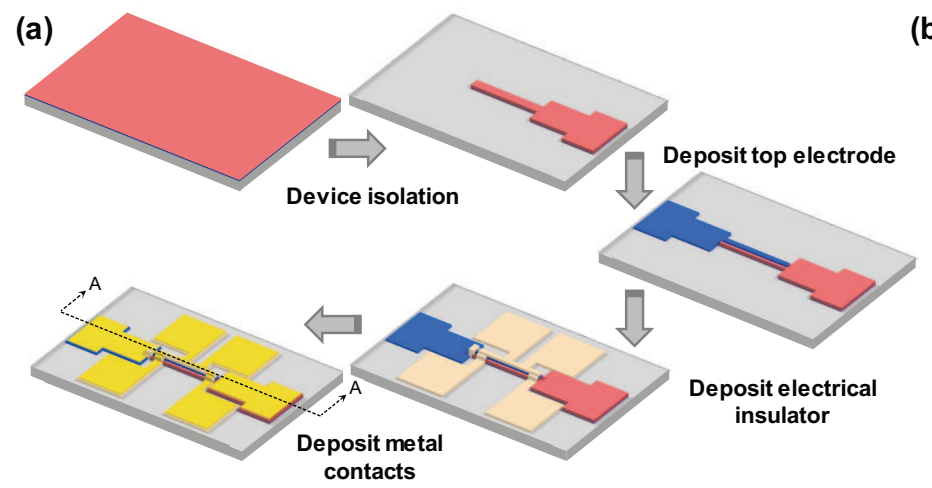

(b)

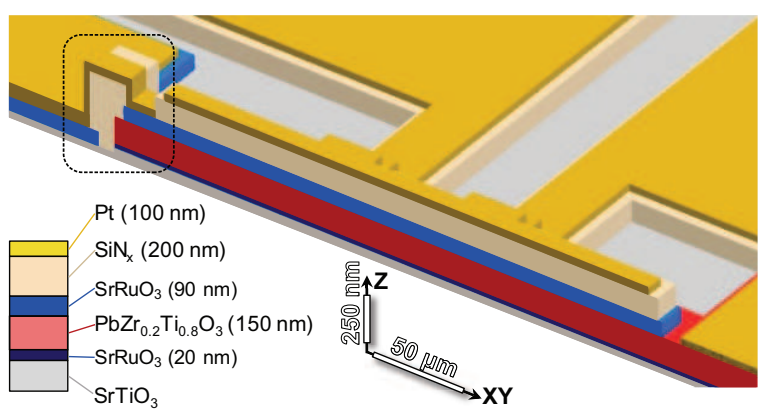

Figure 1. (a) Schematic of the process to fabricate the electrothermal device for measuring pyroelectric and electrocaloric effects in thin films. (b) Section-view through the view A-A depicting various layers in the heterostructure. The film under test here is $\mathrm{PbZr}_{0.2} \mathrm{Ti}_{0.8} \mathrm{O}_{3}$ (red), while the top and the bottom $\mathrm{SrRuO}_{3}$ layers (blue and dark blue, respectively) connect to the top and bottom metal contacts.

to the fact that considerably higher fields can be applied to these thin versions of materials. [23] In turn, reports of large ECE in thin films of $\mathrm{PbZr}_{0.95} \mathrm{Ti}_{0.05} \mathrm{O}_{3}, 24$ (1x) $\mathrm{PbMg}_{y} \mathrm{Nb}_{1-y} \mathrm{O}_{3}-(\mathrm{x}) \mathrm{PbTiO}_{3},[25,26]$ and $\mathrm{SrBi}_{2} \mathrm{Ta}_{2} \mathrm{O}_{9}$ 27] have rejuvenated interest in the study of ECE.

Despite this growing interest, these electrothermal responses (i.e., PEE and ECE) in thin films remain considerably under-studied compared to dielectric, piezoelectric, and ferroelectric effects. This discrepancy in study (and, in turn, understanding) is primarily related to the fact that direct (and accurate) measurements of the temperature changes are difficult. The small thermal mass of thin-film-based devices and the presence of the substrate leads to non-adiabatic conditions where the film loses heat to the substrate with a time constant smaller than the characteristic time constant for the application (or removal) of the electric field. 28] These inherent difficulties in measurement have, therefore, resulted in the majority of work to date relying on so-called "indirect" methods to measure ECEs. In such indirect approaches, the polarization is measured as a function of electric field at different temperatures to extract $\left(\frac{\partial P}{\partial T}\right)_{E}$ and the ECE is inferred via application of the Maxwell relation $\left(\frac{\partial P}{\partial T}\right)_{E, \sigma o r \varepsilon}=\left(\frac{\partial S}{\partial E}\right)_{T, \sigma o r \varepsilon}$. This allows one to estimate the isothermal dipolar entropy change $\left(\Delta S_{i s o}\right)$ and, in turn, the adiabatic temperature change $\left(\Delta T_{a d}\right)$ as $[2,29]$

$$
\begin{array}{r}
\Delta S_{i s o}=\int_{E_{1}}^{E_{2}}\left(\frac{\partial P}{\partial T}\right)_{E} d E \\
\Delta T_{a d}=-\int_{E_{1}}^{E_{2}}\left(\frac{T}{C(T, E)}\right)\left(\frac{\partial P}{\partial T}\right)_{E} d E .
\end{array}
$$

where $C(T, E)$ is the volumetric heat capacity. Many recent reports of large ECE, including $\Delta T=12 \mathrm{~K}$ for $\mathrm{PbZr}_{0.95} \mathrm{Ti}_{0.05} \mathrm{O}_{3}\left(\Delta E=480 \mathrm{kV} \mathrm{cm}{ }^{-1}\right.$, initial temperature of $\left.220^{\circ} \mathrm{C}\right),[24] \Delta T \approx 12 \mathrm{~K}$ for the ferroelectric copolymer $\mathrm{P}(\mathrm{VDF}-\mathrm{TrFE}),[30]$ and colossal $\Delta T=45.3 \mathrm{~K}$ at the transition temperature of films of the relaxor ferroelectric $\mathrm{Pb}_{0.8} \mathrm{Ba}_{0.2} \mathrm{ZnO}_{3}[31$ have utilized this indirect approach. Correct application of this approach, however, requires that the appropriate mechanical boundary conditions of the material are considered; particularly in the case of thin films where the mechanical clamping to the rigid substrate breaks the thermodynamic equivalence of $\Sigma$ and $\pi$. 32] Ultimately, the development of reliable measurement techniques for thin films is needed to truly understand the mechanism and magnitude of these responses and to inspire more confidence for the design of electrocaloric devices.

In this work, we develop an electrothermal test platform to characterize the PEE and ECE in thin films using an adaptation of "2-omega" and "3-omega" techniques, respectively. Traditionally, such 3-omega approaches have been used to measure the thermal conductivity of thin films. [33] For measurements of the PEE, the ferroelectric thin film is locally heated in a periodic fashion via a thin-film, microfabricated heater at frequencies up to $\sim$ $1 \mathrm{kHz}$ and the resulting current is measured using phasesensitive detection. Using the 3-omega method, periodic temperature oscillations in the ferroelectric result from periodic heating of the heater line is measured. In turn, by measuring the thermal phase lag in the ferroelectric layer, the thermally-stimulated current (which lags the pyroelectric current by $90^{\circ}$ ) are separated from the pyroelectric current offering accurate probes of PEE. The same device can then further be used to measure directly the ECE in thin films. In the ECE measurement, AC electric fields are applied across the ferroelectric capacitor and the resulting temperature change of the top microfabricated heater (now functioning solely as a temperature sensor) is measured using a modified 3-omega method. Using 1D analytical heat transport models, the $\Sigma$ and the average temperature change in the ferroelectric is calculated. Measurement of both PEE and ECE on the same device allows us to compare the differences between the two electrothermal effects. Here, we describe the de- 



Figure 2. (a) DC Current-voltage characteristics, (b) ferroelectric hysteresis loops, and (c) dielectric permittivity and loss tangent as a function of frequency for a $150 \mathrm{~nm}$ thick $\mathrm{PbZr}_{0.2} \mathrm{Ti}_{0.8} \mathrm{O}_{3}$ device obtained at $300 \mathrm{~K}$.

sign and fabrication of these electrothermal devices, the underlying physics of the measurements and the extraction of the physical properties, and demonstrate the capabilities of this technique in a model ferroelectric thin film of $\mathrm{PbZr}_{0.2} \mathrm{Ti}_{0.8} \mathrm{O}_{3}$. We will show that pyrocurrents $<10 \mathrm{pA}$ and temperature changes $<2 \mathrm{mK}$ can be reliably measured allowing us to characterize micron-scale, thin-film $(\sim 100 \mathrm{~nm})$ devices with extremely small thermal mass $\left(\sim 6 \times 10^{-9} \mathrm{~g}\right)$.

\section{DEVICE DESIGN}

Measurement of both the PEE and ECE on the same thin-film heterostructure requires the ability to 1 ) heat the ferroelectric thin film and measure $i_{P}$ flowing across the ferroelectric in a capacitor circuit, and 2) to apply electric fields across the ferroelectric and measure the temperature change in the ferroelectric layer. This calls for an independent ferroelectric circuit, comprised of a rectangular capacitor-like geometry with symmetric top and bottom electrodes, and a thermal circuit, comprised of a narrow thin-film metal strip acting as a heater wire and four-point probes for thermal sensing.

Here, as a demonstration of the potential of this approach, we focus on measuring the electrothermal response of $150 \mathrm{~nm}$ thin film of $\mathrm{PbZr}_{0.2} \mathrm{Ti}_{0.8} \mathrm{O}_{3}$ epitaxially grown on $20 \mathrm{~nm} \mathrm{SrRuO}_{3} / \mathrm{SrTiO}_{3}$ (001) via pulsedlaser deposition using established processes. 34] Following growth, the electrothermal characterization devices are produced via a multi-step, micro-fabrication process. Briefly, the ferroelectric heterostructure is lithographically patterned and ion-milled to define the bottom electrode and the ferroelectric "active" layer (Figure 1a). This step removes the bottom electrode $\left(\mathrm{SrRuO}_{3}\right)$ everywhere except under the active layer and the bottom electrode probe pad. This greatly reduces the risk of electrical shorting of the top and the bottom electrode during wire bonding. In this work, $90 \mathrm{~nm} \mathrm{SrRuO}_{3}$ is then selectively deposited as a symmetric top electrode using a $\mathrm{MgO}$ hard mask process 35] to establish a rectan- gular ferroelectric capacitor geometry $(300 \mu m \times 20 \mu m)$. It should be noted that the $\mathrm{SrRuO}_{3}$ deposited over the ferroelectric/bottom electrode mesa does not contact the $\mathrm{SrRuO}_{3}$ deposited on the ion-milled region of the substrate to be used later for establishing an electrical contact pad. This is done purposefully to ensure that the $\mathrm{SrRuO}_{3}$ does not get deposited on the sidewalls of the ferroelectric layer and electrically short to the bottom electrode. Next, to electrically isolate this ferroelectric circuit from the thermal circuit, a $200 \mathrm{~nm}$ thick blanket layer of $\mathrm{SiN}_{x}$ is deposited using plasma-enhanced chemical vapor deposition $\left(\mathrm{SiH}_{4}+\mathrm{NH}_{3}\right.$ based). $\mathrm{SiN}_{x}$ was chosen as it exhibits low dielectric constant $(\approx 7)$ and a thermal conductivity of $2 \mathrm{Wm}^{-1} \mathrm{~K}^{-1}$ measured via the differential 3-omega method. 33] The deposition temperature of the nitride was limited to $350^{\circ} \mathrm{C}$ to limit decomposition (i.e., $\mathrm{Pb}, \mathrm{Ru}$, or $\mathrm{O}$ loss) of the films. This nitride layer was subsequently patterned and selectively etched using reactive ion etching in a $\mathrm{SF}_{6}$ plasma, to obtain an electrically-insulating film on top of the ferroelectric capacitor. Etching the $\mathrm{SiN}_{x}$ from everywhere around the ferroelectric capacitor reduces lateral dissipation of heat which makes the top sensor line more sensitive to measuring small temperature changes. Finally, a $100 \mathrm{~nm}$ thick platinum thin-film resistance heater/thermometer is lithographically patterned in the shape of a thin strip with four probe pads (two outer and two inner pads, see geometry in Figure 1a) to define the thermal circuit (Figure 1b). Platinum contact pads for the top and the bottom electrode are also defined in this step. While the platinum pad for the bottom electrode contacts the bottom $\mathrm{SrRuO}_{3}$ as the device gets wire bonded, the platinum pad for the top electrode contact runs over the $\mathrm{SiN}_{x}$ to contact the top $\mathrm{SrRuO}_{3}$ without shorting with the bottom electrode (see highlighted region in Figure 1b).

Prior to any electrothermal measurements, we investigated the room-temperature current-voltage, ferroelectric, and dielectric response of the thin-film heterostructures. Current-voltage measurements reveal that the devices exhibit symmetric and highly-resistive response $\left(R_{F E}>10^{8} \mathrm{Ohms}\right)$ and that the electrode contacts 

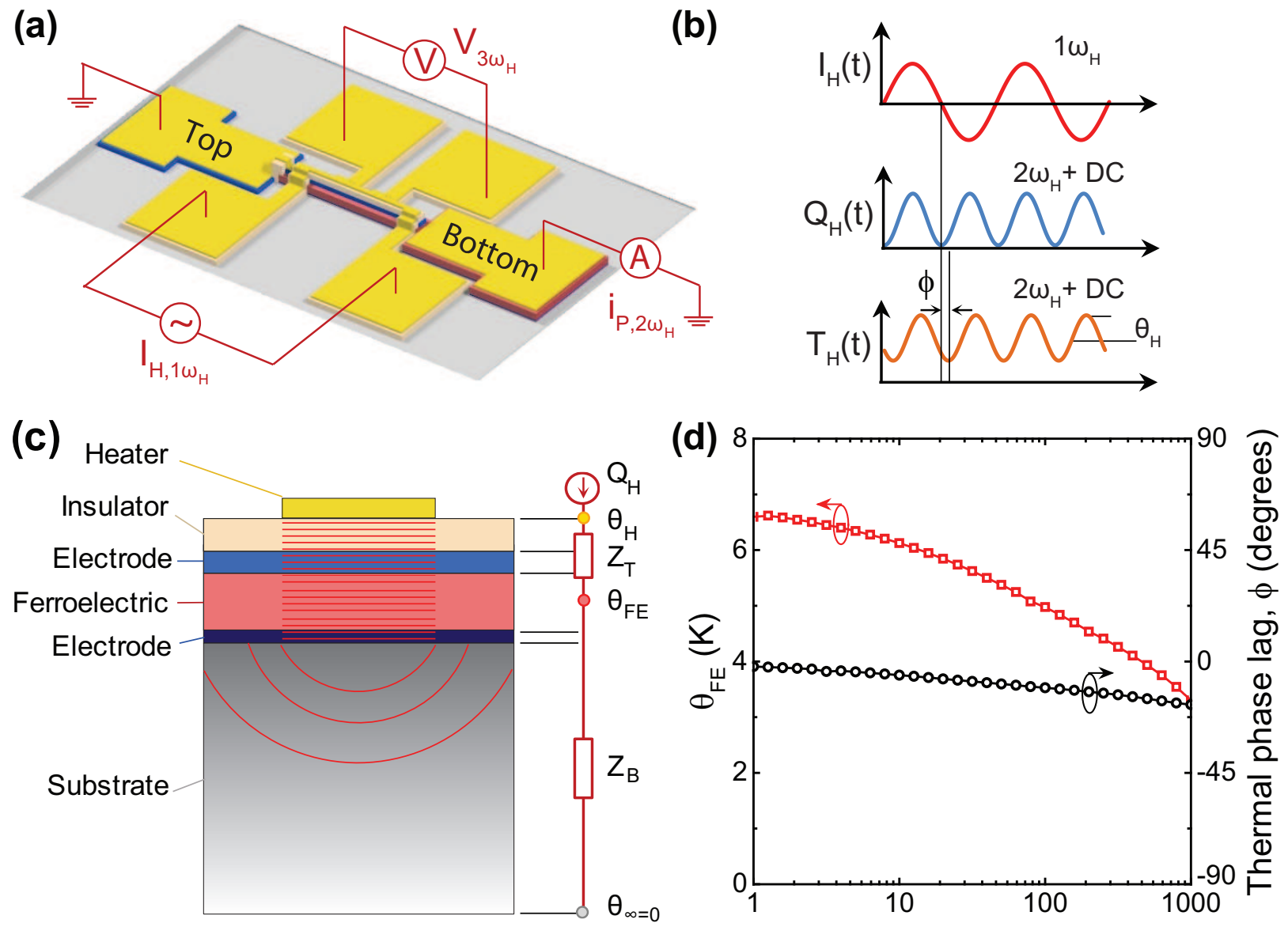

Electrical frequency, $\omega_{\mathrm{H}} / 2 \pi(\mathrm{Hz})$

Figure 3. (a) Schematic of the setup for pyroelectric and 3-omega measurement. (b) Schematic of the relationship between the applied heating current $\left[I_{H}(t)\right]$ at angular frequency $1 \omega_{H}$, dissipated power $\left[Q_{H}(t)\right]$, and the resulting temperature oscillation $\left[T_{H}(t)\right]$ of the metal heater line at $2 \omega_{H}$. (c) Schematic of the temperature field during a pyroelectric measurement and a simplified thermal circuit.(d) Measured temperature oscillation $\theta_{F E}$ and thermal phase lag $\phi$ between the heating current (and, therefore, the dissipated power) and the temperature change in the ferroelectric as a function of frequency.

are rectifying; [36] altogether confirming the high-quality, low-leakage characteristics of the heterostructures (Figure 2a). Ferroelectric hysteresis loops were measured between 0.033 and $1000 \mathrm{~Hz}$ (Figure 2b) and the devices exhibit robust ferroelectric response with low-loss, square ferroelectric hysteresis loops, large remnant polarization $\left(\mathrm{P}_{r} \approx 77 \mu \mathrm{C} / \mathrm{cm}^{2}\right)$, and loop closure even at the lowest measurement frequencies; again confirming the excellent quality of the ferroelectric films and devices. Low-field dielectric permittivity measurements were also conducted on the same devices (Figure 2c). A near-frequencyindependent value of dielectric permittivity $(\sim 248 \pm 2.7)$ was measured across the frequency range of $10^{2}-10^{5} \mathrm{~Hz}$ at room temperature; consistent with expectations for these heterostructures. 37.

\section{THEORY OF MEASUREMENTS}

\section{A. Pyroelectric effect}

The pyroelectric measurement (Figure 3a) relies on the application of a sinusoidally-varying current $\left[I_{H, 1 \omega_{H}}=I_{0 H} \cos \left(\omega_{H} t\right)\right]$ at frequency $\omega_{H}$ across the outer thermal probe pads. The driving current at frequency $\omega_{H}$ dissipates power equal to $I_{H, 1 \omega_{H}}^{2} R_{0}=$ $\frac{1}{2} I_{0 H}^{2}\left[1+\cos \left(2 \omega_{H} t\right)\right]$, where $R_{0}$ is the resistance of the heater line $(\sim 30 \mathrm{Ohms})$. Because the response in the thermal domain is linear, periodic Joule heating causes temperature oscillations with AC amplitude $\theta_{H}$ at $2 \omega_{H}$ (Figure 3b). For a simple heater-on-substrate geometry, the heat generated in the top metal heater line diffuses into the substrate a characteristic distance (often referred to as penetration depth or thermal wavelength) defined as 38 


$$
\lambda_{S}=\sqrt{\frac{D_{S}}{2 \omega_{H}}}
$$

where $D_{S}$ is the thermal diffusivity of the substrate.

To calculate the $\mathrm{AC}$ amplitude of temperature oscillation in the ferroelectric thin film $\left(\theta_{F E}\right)$, first the amplitude of the temperature change in the heater line $\left(\theta_{H}\right)$ was measured using the 3 -omega method. 33] The heating frequency was chosen so that the heat completely propagates through the film stack while still ensuring that the substrate remains semi-infinite such that the thermal waves completely dampen within the substrate (Figure 3c). Thus, the natural bounds on the thermal wavelength are $\lambda_{F E}>3 d_{F E}$ and $\lambda_{S}<\frac{1}{3} d_{S}$, where the subscripts $F E$ and $S$ denote the ferroelectric film and substrate, respectively, and $d$ is the thickness of the relevant component. Key to the sensitivity and accuracy of this approach is the fact that the thermal impedance of the top thermal circuit $Z_{T}$ (comprising the heater line, the electrical insulator and the top oxide metal electrode) is much smaller than that of the bottom circuit $Z_{B}$ (comprising the bottom oxide metal electrode and the substrate), thereby minimizing the error between $\theta_{H}$ and $\theta_{F E}$. The amplitude and phase $(\phi)$ of the temperature oscillation of the heater line depend upon the thermal properties of the system (i.e., thermal conductivity and specific heat capacity) and are measured using the 3omega method.

The measured $\theta_{H}$ and the thermal flux $\left(q_{H}\right)$ into the heterostructure, (i.e., the AC power dissipated per unit area of the heater), are used with a one-dimensional thermal transport model [39] to calculate $\theta_{F E}$ (see Appendix A for details). This scheme is applied to calculate $\theta_{F E}$ at five equally-spaced nodes within the ferroelectric layer. We found that the difference in the temperature amplitude between the top (just below the ferroelectric and top electrode interface) and bottom nodes (just above the ferroelectric and bottom electrode interface) corresponds to $1.6 \%$ of the average $\theta_{F E}$. Hence, any tertiary PEE [40] induced by temperature gradients in the film can be neglected and we can use the lumped parameter approach where the ferroelectric layer is considered to be isothermal at any instant. A similar approach [16] calculates $\theta_{F E}$ using $q_{H}$ and works from the bottom of the substrate up to the ferroelectric layer. We, however chose to work from the top down so as to maximize the number of measured quantities in the model to compensate for any unideal deviation from theory.

The mean temperature oscillation of the ferroelectric, averaged across the five nodes, $\theta_{F E}$, as a function of frequency is thus extracted and plotted (Figure 3d). It can be seen that $\theta_{F E}$ decreases and the phase between the dissipated power (as a result of heating) and the temperature $(\phi)$ increasingly lags as the frequency of $\mathrm{AC}$ heating current on the top metal line increases (Figure $3 \mathrm{~d})$. The phase lag behaves as expected, approaching $0^{\circ}$ and $-17.5^{\circ}$ in the low and high frequency limits, respec- tively, reflecting the gradual transition from cylindrical to one-dimensional heat transport. Determination of thermal phase lag is particularly important for extracting $i_{P}$ which can be convoluted with thermally-stimulated currents that are in-phase with the temperature change.

With the known thermal state of the heterostructure in response to periodic Joule heating, we can proceed to extract $i_{P}$ and $\pi$. The current is measured through the bottom electrode using the current input $\left(10^{6} \mathrm{~V} / \mathrm{A}\right.$ gain $)$ of a lock-in amplifier (Stanford Research, SR830) (Figure 3a). The top electrode is held at ground potential which shields any capacitive coupling between the heating and the ferroelectric measurement circuits. Since $i_{P}$ is proportional to the rate of change of temperature, it leads the temperature change by $90^{\circ}$, and is extracted by taking the component of the measured total current which is out-of-phase (leads by $90^{\circ}$ ) with the temperature change. [14, 15] Thereafter, $\pi$ can be calculated using the formula, $i_{P}=A \pi \frac{d T}{d t}$.

\section{B. Electrocaloric effect}

Measuring the ECE relies on the ability to sense small temperature changes when an electric field is applied and removed across the ferroelectric. A sinusoidally-varying electric field, $E=\left(\frac{V_{0}}{d_{F E}}\right) \sin \left(\omega_{E} t\right)$, when applied to the bottom electrode of the ferroelectric capacitor as depicted (Figure 4a), results in an adiabatic temperature change via the ECE

$$
\Delta T_{a d}=-\int_{E_{1}}^{E_{2}}\left(\frac{T}{C(T, E)}\right)_{E} \Sigma d E
$$

The temperature change via the ECE, unlike the case of Joule heating which happens at DC and $2 \omega$, happens at $\omega$. This can be understood by calculating the ECE power $\left(Q_{E}\right)$

$$
Q_{E}=C(T, E) A d_{F E} \frac{d T}{d t}
$$

where $\mathrm{A}$ is the area of the heat source (ferroelectric layer). Using equation (4), equation (5) can be simplified to

$$
Q_{E}=-T \Sigma A \omega_{E} V_{0 E} \cos \left(\omega_{E} t\right)
$$

Hence, the electrocaloric power and the resultant temperature change occur at the same frequency, $\omega_{E}$ (Figure 4b). Power dissipation due to Joule heating on the other hand is given by

$$
Q_{J}=\frac{V_{0 E}^{2}}{2 R_{F E}}\left(1-\cos \left(2 \omega_{E} t\right)\right)
$$




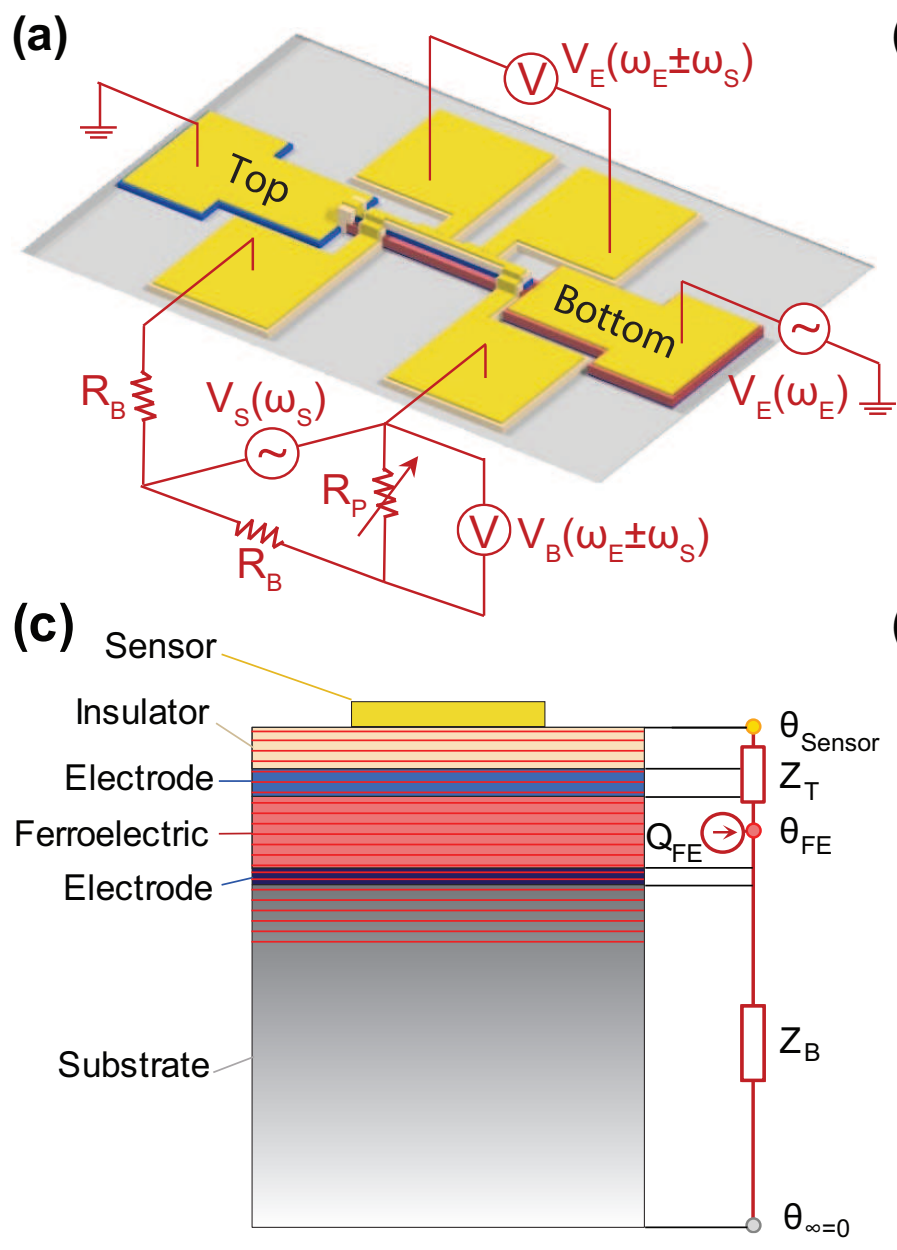

(b)
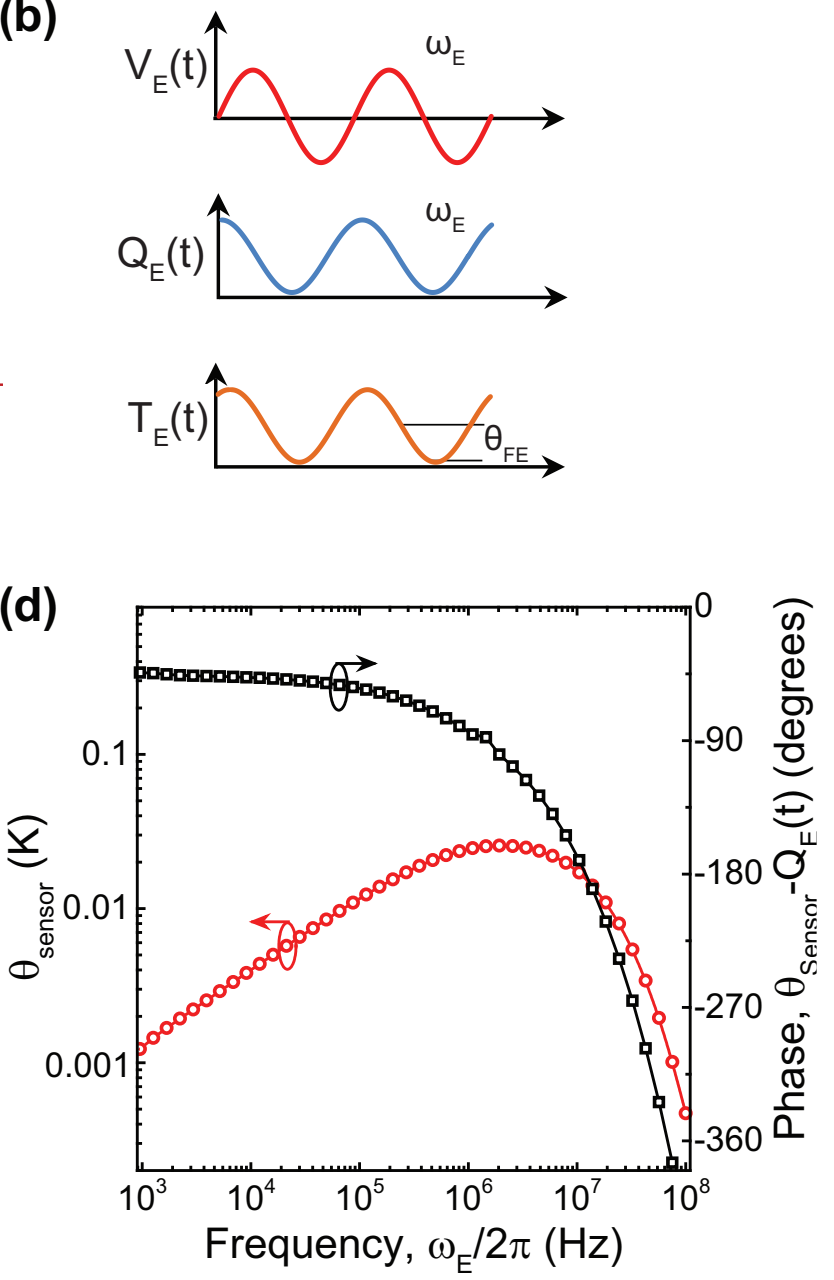

Figure 4. (a) Schematic of the setup for electrocaloric measurement using the top metal line as a resistance thermometer (sensor). (b) Schematic of the relationship between the applied voltage $\left[V_{E}(t)\right]$ across the ferroelectric capacitor at angular frequency $\omega_{E}$, the power generated $\left[Q_{E}(t)\right]$, and the resulting temperature change $\left[T_{E}(t)\right]$ due to the ECE at the same frequency $\omega_{E}$. (c) Schematic of the temperature field during an electrocaloric measurement. (d) Computed temperature oscillation $\theta_{\text {Sensor }}$ and thermal phase lag between the temperature oscillation of the top sensing metal line and the ECE power as a function of frequency of voltage cycling across the ferroelectric for an assumed $\Sigma=-100 \mu \mathrm{Cm}^{-2} \mathrm{~K}^{-1}$ and an applied electric field amplitude of $50 \mathrm{kVcm}^{-1}$.

where $R_{F E}$ is the resistance of the ferroelectric. Thus, Joule heating still happens at DC and $2 \omega_{E}$ thereby making ECE measurements in the frequency-domain at $\omega_{E}$ effective in filtering out contributions from Joule heating.

The temperature oscillation of the top sensor line $\left(\theta_{\text {Sensor }}\right)$ can be related to the ECE power from the underlying ferroelectric film $\left(Q_{E}\right)$ via a generic thermal transfer function in the frequency domain, $Z$ [41]

$$
\theta_{\text {Sensor }}(t)=Q_{0 E}\left[\operatorname{Re}(Z) \cos \left(\omega_{E} t\right)+\operatorname{Im}(Z) \sin \left(\omega_{E} t\right)\right]
$$

where $Q_{0 E}=-T \Sigma A \omega_{E} V_{0 E}$ from equation (6). This temperature change perturbs the electrical resistance of the top metal heater/sensor line as

$$
R_{\text {Sensor }}=R_{0, \text { Sensor }}\left[1+\alpha \Delta T_{D C}+\alpha \theta_{\text {Sensor }}\right]
$$

where $R_{0, \text { Sensor }}$ is the resistance of the sensor line at 300 $\mathrm{K}(\sim 10 \mathrm{Ohms})$ and $\alpha$ is the temperature coefficient of resistance. To sense the temperature in the top metal line, a sensing signal of the form $V_{S} \sin \left(\omega_{S} t\right)$ is applied across the outer thermal pads (Figure 4a). The voltage source is converted to a current source by inclusion of a ballast resistor $\left(R_{B}\right.$, which is $10^{2}$ times larger than $\left.R_{\text {Sensor }}\right)$ in series. Other approaches include the use of a commercially-available AC current source (for example, Keithley $6221 \mathrm{~A}$ ) or a custom-built V-to-I circuit using operational amplifiers. 42] Voltage $\left(\mathrm{V}_{A}\right)$ is measured across the inner pads of the thermometer and has a form 


$$
V_{A}=\frac{V_{S} \sin \left(\omega_{S} t\right)}{R_{B}+R_{\text {Sensor }}} R_{\text {Sensor }}
$$

Since $\frac{R_{B}}{R_{\text {Sensor }}} \geq 10^{2}$, using equation (8) and (9), equation (10) can be expressed as

$$
\begin{gathered}
V_{A}=\frac{V_{S} \sin \left(\omega_{S} t\right)}{R_{B}} R_{S e n s o r}\left[1+\alpha \Delta T_{D C}+\right. \\
\left.\alpha Q_{0 E}\left(\operatorname{Re}(Z) \cos \left(\omega_{E} t\right)+\operatorname{Im}(Z) \sin \left(\omega_{E} t\right)\right)\right]
\end{gathered}
$$

The response frequencies $\omega_{S} \pm \omega_{E}$ (due to the product of two sinusoidal functions) contain the information regarding the temperature oscillation of the sensor. The voltage signal corresponding to $\omega_{S}$ is particularly large and can be 1000-times larger than the one corresponding to $\omega_{S} \pm \omega_{E}$ resulting in a significantly larger background. Therefore, a common mode cancellation circuit using a Wheatstone bridge has been implemented in our measurement scheme (Figure 4a). The sensing signal is simultaneously applied across a potentiometer which is adjusted such that the resistance of the potentiometer $R_{P} \approx R_{0 \text { Sensor }}$ and, therefore, the voltage drop $\left(V_{B}\right)$ across $R_{P}$ nullifies the $\omega_{S}$ component of the differential voltage $\left(V_{A}-V_{B}\right)$ that is fed to the lock-in amplifier. Note that the $\omega_{S} \pm \omega_{E}$ signal only comes from the temperaturedependent response due to the ECE $\left(R_{\text {Sensor }}\right)$ and is absent in the potentiometer $\left(R_{P}\right)$. Therefore, only the $\omega_{S}$ background is attenuated and the measurement of the much smaller $\omega_{S} \pm \omega_{E}$ signal becomes possible.

The measured differential voltage $\left(V_{\omega_{S} \pm \omega_{E}}\right)$ is related to the temperature oscillation of the top sensor via [43, 44]

$$
\theta_{\text {Sensor }}=2 \frac{1}{\alpha V_{\omega_{S}}} V_{\omega_{S} \pm \omega_{E}}
$$

This temperature change of the top sensor line is used to calculate $\Sigma$ as well as the average temperature oscillation in the ferroelectric, $\theta_{F E}$. This requires knowledge of the volumetric power generation $(G)$ created by the entropy change and can be related to $\Sigma$ as 32 ]

$$
G=-T \frac{d S}{d E} \frac{d E}{d t}=-T \Sigma\left(\frac{V_{0 E}}{d_{F E}}\right) \omega_{E} \cos \left(\omega_{E} t\right)
$$

The resulting heat flux can be determined from the measured temperature change on the top sensor line and the thermal properties of all the layers in the heterostructure. Due to the ECE, heat flux is generated homogeneously in the ferroelectric and diffuses in both directions (i.e., into the substrate and into the top sensor line). To model this heat transport, we solve a $1 \mathrm{D}$ problem for the heat flux generated in each differential thickness $(d z)$ at a height $z$ in the ferroelectric. [32] We defined $G$ to be the power generated per unit volume and note the differential flux $d q_{t o t}$ generated from each volume is $d q_{t o t}=G d z$.
The total temperature oscillation sensed by the metal sensor is the sum of the contributions from the entire thickness of the ferroelectric (Figure 4c). This is a continuous domain, so summation is replaced by integration and the amplitude of the temperature oscillations sensed by the metal line is defined by

$$
\theta_{\text {Sensor }}=G \int_{0}^{d_{F E}} \xi d z
$$

where $\xi$ is an algebraic expression calculated using the elements of the thermal transport matrices and the appropriate boundary conditions (see Appendix B for details). Using equation (13), equation (14) simplifies to

$$
\theta_{\text {Sensor }}=-T \Sigma\left(\frac{V_{0 E}}{d_{F E}}\right) \omega_{E} \cos \left(\omega_{E} t\right) \int_{0}^{d_{F E}} \xi d z
$$

Equation (15) allows the calculation of $\Sigma$ from the measured value of $\theta_{\text {Sensor }}$. Further, the average temperature oscillations of the ferroelectric, $\theta_{F E}$, can be calculated using a similar approach to find the temperature amplitudes at the top and bottom surface of the ferroelectric then taking their average.

In our measurements, high-frequency electric field oscillations are required to generate enough flux to produce measurable temperature change. The effect of driving frequency on the resulting temperature change on the top sensor line, for an assumed $\Sigma=-100 \mu \mathrm{Cm}^{-2} \mathrm{~K}^{-1}$ and an applied electric field oscillation of $50 \mathrm{kVcm}^{-1}$, is calculated from the above thermal models and shown for reference (Figure 4d). It can be seen that at lower frequencies $\left(<3 \times 10^{6} \mathrm{~Hz}\right)$, the temperature change on the top metal sensor is limited due to low thermal flux [refer to equation (15)]. At high frequencies $\left(>3 \times 10^{6}\right.$ $\mathrm{Hz}$ ), the temperature change diminishes due to the limited thermal penetration depth, placing an upper limit on the frequency range of the experiment. It is therefore desirable to perform ECE measurements at frequencies in the range close to $2 \times 10^{5}-1 \times 10^{6} \mathrm{~Hz}$.

\section{RESULTS AND DISCUSSION}

\section{A. Pyroelectric effect}

Following the theory of pyroelectric measurement in section IIIA (Figure 3), the power spectrum of the measured total current $\left(i_{\text {Total }}\right)$ is provided (Figure $5 \mathrm{a}$ ) for a heating current $\left(I_{H, 1 \omega_{H}}\right)$ equal to $16 \mathrm{~mA}$ at $1 \mathrm{kHz}$. It can be seen that a dominant current peak occurs at frequency $2 \omega_{H}$ as a result of the temperature oscillations at the same frequency in the ferroelectric due to the driving current at $1 \omega_{H}$ in the heater line (inset, Figure 5a). It should be noted that the presence of the electricallyinsulating $\mathrm{SiN}_{x}$ layer and electrical shielding via the top electrode reduce, but do not completely suppress, the 
capacitive coupling. This manifests as the observed response at $\omega_{H}$ (Figure 5a); however, our ability to measure $i_{P}$ (measured at $2 \omega_{H}$ frequency) is not affected by the non-zero capacitive coupling. The basis of this argument is twofold. First, measurements with zero heating, but with capacitive bias across the heterostructure, yield $2 \omega$ current which is $\sim 10^{3}$ smaller than the case when heating occurs in conjunction with the capacitive bias. Second, we measured the total current with the polarization of the ferroelectric in the up-poled state $\left(i_{u p}\right)$ and the down-poled state $\left(i_{\text {down }}\right)$. Since the switching of the polarization only reverses the direction of $i_{P}$ (manifested as a phase reversal by $180^{\circ}$ ) and does not change the capacitively-coupled parasitic current, $i_{u p}$ and $i_{\text {down }}$ can be expressed as

$$
\begin{aligned}
i_{u p} & =i_{P}+i_{\text {Parasitic }}, \\
i_{\text {down }} & =-i_{P}+i_{\text {Parasitic }}
\end{aligned}
$$

By taking the sum and difference of equations (16) and (17), we are able to subtract out any contribution due to parasitic-coupling which constitutes less than $4 \%$ of the total measured current. These two checks confirmed that the current due to parasitic capacitance is negligible. This, however, does not prove that the current is completely pyroelectric in origin. Our measurements show that the measured current leads the actual temperature change in the ferroelectric (Figure $3 \mathrm{~d}$ ) by $\sim 90^{\circ}$ (Figure 5b). Deviations from the expected phase can be attributed to thermally-stimulated currents which flow in-phase (zero lag) with the temperature change. The real $i_{P}$ is extracted by taking the component of the total current which is out-of-phase with the temperature change and is plotted as a function of the frequency of heating current (red circles, Figure 5c). Apparently $\pi$ has a frequency dependence (black squares, Figure 5c). At low frequencies $(<4 \mathrm{~Hz}), \pi$ is seen to begin to converge to a value of $\sim-280 \mu \mathrm{Cm}^{-2} \mathrm{~K}^{-1}$, while at higher frequencies $(>500 \mathrm{~Hz})$ it asymptotes to a lower value of $\sim-195 \mu \mathrm{Cm}^{-2} \mathrm{~K}^{-1}$. This reduction $(\sim 30 \%)$ can be explained by the secondary contribution 45 to the total pyroelectricity due to the thermal expansion mismatch between film and substrate and the resulting thermalstress-induced, piezoelectric-driven polarization change. At low frequency, the thermal penetration depth $\left(\lambda_{S}\right)$ is large compared to the lateral dimensions of the device, therefore, the ferroelectric film expands and contracts inplane with the substrate. As a result, the total $\pi$ under a constant stress boundary condition can be expressed as

$\pi=\left(\frac{\partial P}{\partial T}\right)_{E, \sigma}=\left(\frac{\partial P}{\partial T}\right)_{E, \varepsilon}+\left(\frac{\partial P}{\partial \sigma}\right)_{E, T}\left(\frac{\partial \sigma}{\partial \varepsilon}\right)_{E, T}\left(\frac{\partial \varepsilon}{\partial T}\right)_{E, \sigma}$

At higher frequencies, the lateral dimensions of the film do not change as the film is clamped to the substrate which cannot expand laterally due to the reduced thermal penetration depth. Therefore, the contribution from the lateral thermal expansion $\left(\frac{\partial P}{\partial \sigma}\right)_{E, T}\left(\frac{\partial \sigma}{\partial \varepsilon}\right)_{E, T}\left(\frac{\partial \varepsilon}{\partial T}\right)_{E, \sigma}$ is suppressed and the magnitude of $\pi$ decreases.

Changes in temperature not only change the permanent dipole moment (intrinsic portion of primary pyroelectricity), but also alter the dielectric permittivity and hence the surface charge density under an applied electric field. Particularly near a dielectric anomaly (for example, near a phase transition), the dielectric constant changes sharply with temperature and can result in either a significant suppression or enhancement of $i_{P}$ depending upon the poled-state of the ferroelectric. Full characterization of $\pi$ therefore requires one to measure $i_{P}$ as a function of background electric field. In our measurements, this is achieved by applying a constant bias voltage to the top electrode while the bottom electrode remains connected to the input of the lock-in amplifier. The measured pyroelectric hysteresis loops are provided (Figure 5d). When the ferroelectric is up-poled, increasing the temperature results in a "positive" $i_{P}$ (red data, Figure 5d). With increasing positive bias (greater than the coercive field) on the top electrode, the polarization switches and causes $i_{P}$ to reverse its direction (blue data, Figure 5d). When the direction of electric field bias is reversed (i.e., negative bias greater than the coercive field is applied), the polarization switches back and $i_{P}$ reverses direction again, giving a characteristic hysteresis-like pyroelectric loop. It should be noted that the slope of $i_{P}$ vs. electric field is close to zero away from the switching near the coercive fields indicating that the contribution from the temperature-dependent dielectric permittivity is negligible as the dielectric permittivity does not change strongly with temperature far from the transition temperature $\left(T_{C}>450^{\circ} \mathrm{C}\right)$.

\section{B. Electrocaloric effect}

Measurements were performed on the same device on which the pyroelectric measurements were conducted. The frequency $\left(\omega_{E}\right)$ of the applied electric field of magnitude $\left(\frac{V_{0 E}}{d_{F E}}\right)=50 \mathrm{kV} \mathrm{cm}^{-1}$ was chosen to be $98,147 \mathrm{~Hz}$ while that of the sensing voltage $\left(\omega_{S}\right)$ was chosen to be $2,317 \mathrm{~Hz}$ with a magnitude $V_{S}=7.07 \mathrm{~V}$. The choice of frequency was determined so as to avoid any resonance between the signal to be measured and power supply noise (harmonics of $60 \mathrm{~Hz}$ ) or any higher-order harmonics of the sensing signal frequency. A full frequency-domain voltage response is plotted (Figure 6a). As discussed above, the ECE response in the frequency-domain occurs at $\omega_{S}-\omega_{E}$ and $\omega_{S}+\omega_{E}$ corresponding to 95,830 and $100,464 \mathrm{~Hz}$. This can be clearly seen against the low background (insets, Figure 6a). To further validate our technique, we performed the same measurement with varying magnitudes of applied electric field. We note that the magnitude of the maximum electric field never exceeded the coercive field of switching for the ferroelectric so that the ECE response (change in temperature) 


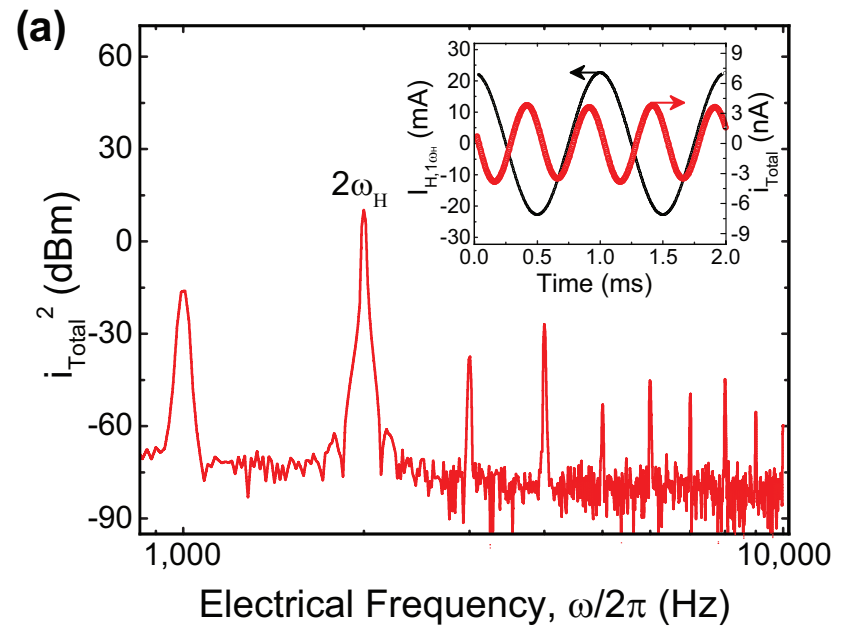

(c)

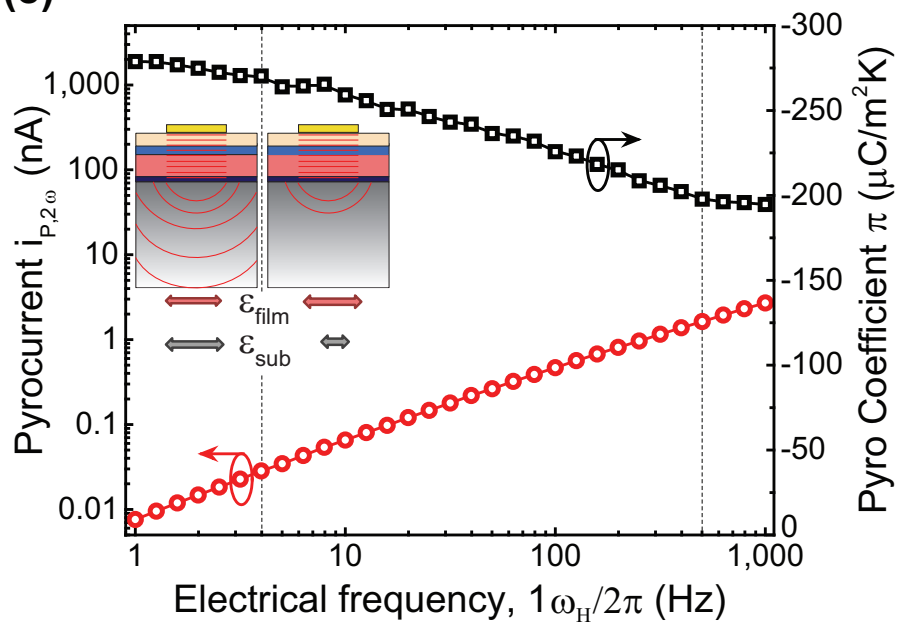

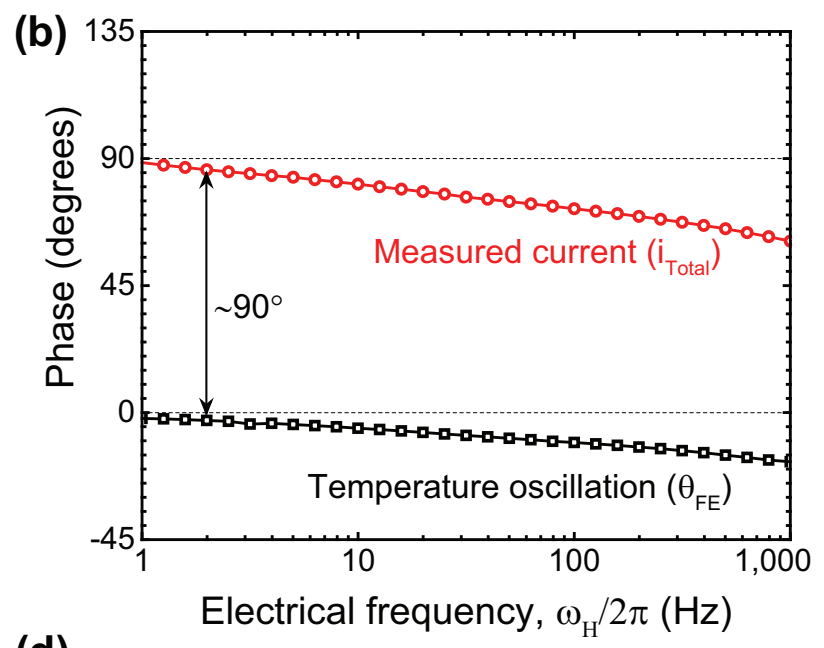

(d)

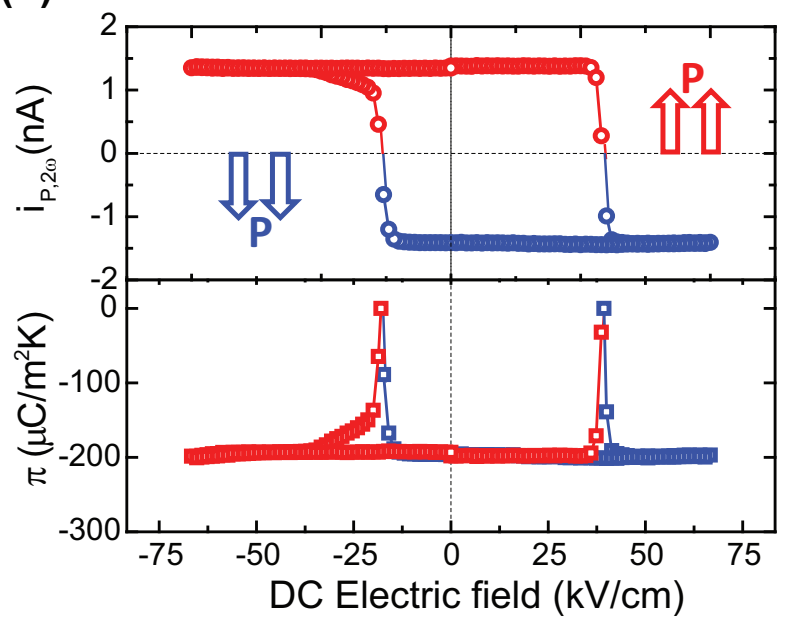

Figure 5. Pyroelectric measurements of $\mathrm{PbZr}_{0.2} \mathrm{Ti}_{0.8} \mathrm{O}_{3}$ thin film. (a) Measured total current $\left(i_{\text {Total }}\right)$ in the frequency-domain and time-domain (inset) for an applied heating current $\left(I_{H, 1 \omega_{H}}\right)$ equal to $16 \mathrm{~mA}$ at a frequency of $1 \mathrm{kHz}$. (b) Phase relationship of the measured current (at $2 \omega_{H}$ ) and the temperature oscillation (also at $2 \omega_{H}$ ) with respect to the phase of the applied heating current depicting that the measured current leads the temperature oscillation by $\sim 90^{\circ}$. (c) Measured pyroelectric current and pyroelectric coefficient as a function of heating frequency. At low frequencies $(<4 \mathrm{~Hz}), \pi \rightarrow\left(\frac{\partial P}{\partial T}\right)_{E, \sigma}$, while at high frequencies $(>500 \mathrm{~Hz}) \pi \rightarrow\left(\frac{\partial P}{\partial T}\right)_{E, \varepsilon}$ due to secondary contribution to pyroelectricity (reduction by $\sim 30 \%$ ). (d) Pyroelectric hysteresis loops as a function of background DC electric field, at $1 \mathrm{kHz}$.

remains linear with applied electric field [refer to equation (15)]. The average temperature change in the ferroelectric layer is found to scale linearly with the applied electric field (red squares, Figure 6b). While frequencyselective measurements at $\omega_{S} \pm \omega_{E}$ inherently remove any Joule heating that happens at $\omega_{S} \pm 2 \omega_{E}$, this measurement additionally proves that the ECE response is free from Joule heating which would have caused $\theta_{F E}$ to scale quadratically with the driving electric field. Using the thermal transport model explained above, $\Sigma$ has been extracted from the measured temperature change of the top metal sensor line (black circles, Figure 6b). A nearfield-independent value of $\Sigma$ is calculated; further proving the linearity of the response in the electric-field regime without any polarization switching. We do observe slight deviations at very low magnitude of electric field which correspond to low signal to noise ratio.

We further measured the ECE response as a function of background DC electric field (Figure 6c), and, similar to the PEE, a hysteresis-like ECE loop is measured. Positive ECE is the increase/decrease in the temperature of the material when an electric field is applied/removed. This is often the case in dielectrics or ferroelectrics above the Curie temperature. Tetragonal ferroelectrics such as $\mathrm{PbZr}_{0.2} \mathrm{Ti}_{0.8} \mathrm{O}_{3}$ below the Curie temperature, however, can exist in either of two stable polarization states. It is the relative direction of the polarization vector and the electric field vector that determines the nature (whether 

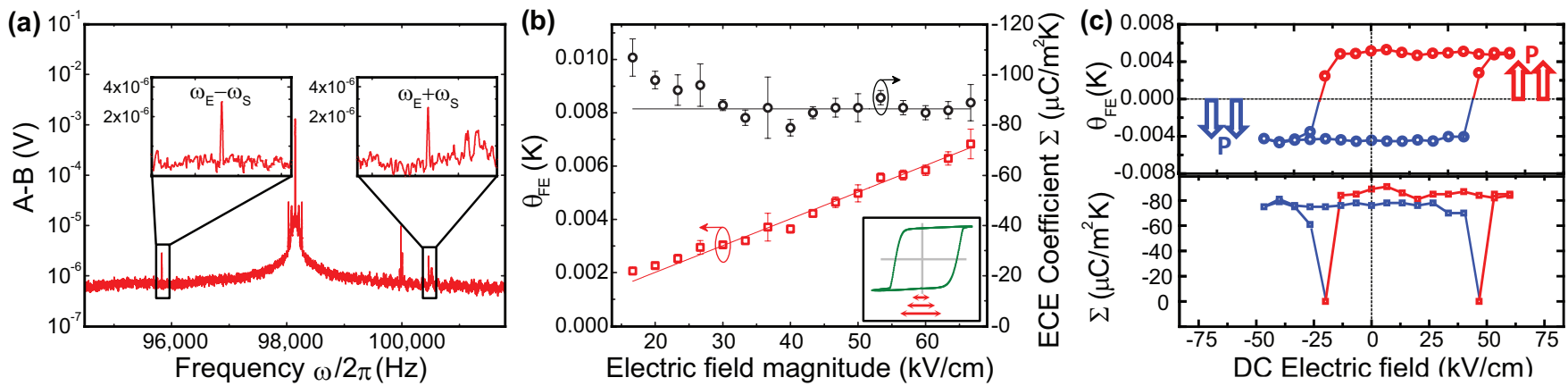

Figure 6. Electrocaloric measurements of $\mathrm{PbZr}_{0.2} \mathrm{Ti}_{0.8} \mathrm{O}_{3}$ thin film. (a) Measured differential voltage across the top metal sensor in the frequency-domain for an applied electric field of magnitude $50 \mathrm{kVcm}^{-1}$ at a frequency $\omega_{E} / 2 \pi$ equal to $98,147 \mathrm{~Hz}$ and a sensing voltage $V_{S}$ equal $7.07 \mathrm{~V}$ at a frequency $\omega_{S} / 2 \pi$ equal to $2,317 \mathrm{~Hz}$. Inset shows the voltage response at frequency $\omega_{S}-\omega_{E}$ and $\omega_{S}+\omega_{E}$ which is a measure of the change in the resistance and thus $\theta_{\text {Sensor }}$ of the top metal. (b) Average measured temperature oscillation in the ferroelectric $\left(\theta_{F E}\right)$ due to the ECE and the resulting calculated value of $\Sigma$. (c) Electrocaloric loops as a function of background DC electric field using an AC electric field amplitude of $50 \mathrm{kVcm}^{-1}$. Up-poled state (red) results in the positive ECE while the down-poled state (blue) results in the negative ECE.

positive or negative) of the ECE. When the ferroelectric is up-poled, applying an electric field parallel to the direction of polarization such that the application of field further reinforces the electrical dipoles results in a positive ECE (red data, Figure 6c). Application of negative electric field greater than the coercive field results in polarization switching. In this down-polarized state, the positive electric field excitation (which is now antiparallel to the direction of polarization) tends to misalign the dipoles thereby increasing the dipolar entropy and hence decreasing the temperature. In this regime, the ECE is negative (blue data, Figure 6c). It is worth mentioning that Joule heating, which under zero background electric field occurs only at DC and $2 \omega_{E}$, has a component at $\omega_{E}$ under a finite background electric field

$$
\begin{array}{r}
Q_{J}=\left(\frac{V_{0 E}^{2}}{2 R_{F E}}+\frac{V_{D C}^{2}}{R_{F E}}\right)+\frac{2 V_{0 E} V_{D C}}{R_{F E}} \sin \left(\omega_{E} t\right) \\
-\frac{V_{0 E}^{2}}{2 R_{F E}} \cos \left(2 \omega_{E}\right)
\end{array}
$$

Should Joule heating be significant, the measured voltage response at $\omega_{S} \pm \omega_{E}$ will have contributions from Joule heating and the shape of the ECE loop will no longer be square-like and the otherwise constant temperature change and $\Sigma$ (zero slope) exhibit a finite slope (proportional to $\left(\frac{2 V_{0 E}}{R_{F E}}\right)$ with increasing temperature change with applied background electric field. Our data (in Figure 6c) does not show any measurable slope under background electric field suggesting a highly insulating device as suggested by the measured electrical properties (Figure 2).

\section{SENSITIVITY OF THE MEASUREMENT}

The thermal model used for the PEE measurements is not sensitive to any material parameter due to the thin layers and low frequencies. Instead, the predominant error in the calculation of the average temperature change in the ferroelectric comes from the calculation of the heater temperature from the 3-omega equations.

To evaluate the accuracy of the ECE measurement due to propagation of errors from the various thermophysical properties, we quantify the sensitivity of the measured temperature response of the top metal line sensor (during ECE measurement) in the thermal model as 46]

$$
S_{x}^{T}=\frac{\partial \ln \theta}{\partial \ln x}
$$

where $x$ is one of the parameters (e.g., thermal conductivity, specific heat capacity, thickness of a particular layer) in the thermal model. With this definition of sensitivity, a value of $S=-0.1$, for example, means that a $10 \%$ increase in parameter $x$ will result in a $1 \%$ decrease in $\theta$. Equation (20) is evaluated for the following layer properties: thermal conductivity $k$, volumetric heat capacity $C$, and layer thickness $d$ as function of frequency (Figure 7). At low frequencies $\left(3<\times 10^{5} \mathrm{~Hz}\right)$, ECE measurements are most sensitive to $k$ and $C$ of the substrate while at high frequency $\left(3>\times 10^{5} \mathrm{~Hz}\right), C$ and $d$ of $\operatorname{SiN}_{x}$ and $\mathrm{PbZr}_{0.2} \mathrm{Ti}_{0.8} \mathrm{O}_{3}$ are the most important parameter.

\section{SUMMARY}

In conclusion, we have demonstrated a reliable technique for direct measurement of the PEE and ECE in thin films using a microfabricated electrothermal test 
ence Foundation under grant P2ELP2-152177.

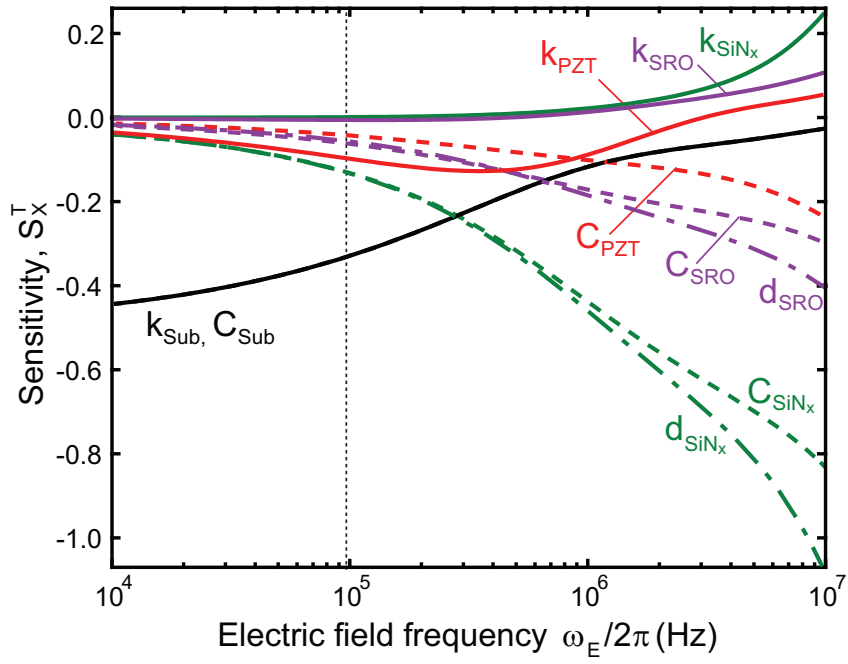

Figure 7. Sensitivity of the calculated electrocaloric temperature change at the top metal sensor, $\theta_{\text {Sensor }}$ as a function of selected parameters in the thermal model as function of frequency of electric field cycling for a $150 \mathrm{~nm} \mathrm{PbZr}_{0.2} \mathrm{Ti}_{0.8} \mathrm{O}_{3}$ heterostructure. The dashed line denotes the frequency of electric-field cycling employed for the electrocaloric experiments.

platform. Periodic heating via the top metal line resulted in localized heating of the ferroelectric and, in turn, in a flow of an external current which was separated into pyroelectric and thermally-stimulated currents using frequency-domain phase-sensitive detection. Further, wide-frequency range measurement depicted the role of the secondary contribution to PEE $(\sim 30 \%)$ due to substrate clamping. The same device was used to measure ECE where high-frequency electric-field oscillation across the ferroelectric resulted in a temperature change which was sensed by the same top metal line now functioning as a resistance thermometer. Frequency-domain measurements allowed decoupling of Joule heating from the ECE. Using 1-D heat transport models, the thermal state of the heterostructure was characterized during the measurement of the PEE and the ECE to extract $\pi$ and $\Sigma$.

\section{ACKNOWLEDGMENTS}

S.P. acknowledge support from the Army Research Office under grant W911NF-14-1-0104. A.R.D. acknowledges support from the National Science Foundation under grant CMMI-1434147. A.D. acknowledges support from the Department of Energy under Grant No. DESC0012375. L.W.M. acknowledges support from the National Science Foundation under grant DMR-1451219. C.M. acknowledges funding from the Swiss National Sci-

\section{Appendix: Description of 1-D heat transport models}

\section{Pyroelectric effect}

The full solution to the heat diffusion equation for a heater-on-substrate geometry is solved in cylindrical coordinates. With the addition of thin films on top of the substrate, it is reasonable to approximate the heat transport through the films as one dimensional so long as the heater half-width, $b(5 \mu \mathrm{m})$, is greater than the total thickness of the film stack $(<0.5 \mu \mathrm{m})$. An exact quantification of the error 38] in making this assumption caps the error in our model at less than $5 \%$.

The steady, periodic solution to the one-dimensional heat diffusion equation is well-described by Carslaw and Jaeger, 39] so many of the details will be skipped here. However, the transport matrix method for practical application of the solution will be described in detail. Starting from the analytical solution to the heat diffusion equation, heat transport across a single domain can be written via vector multiplication as

$$
\left[\begin{array}{l}
\theta_{d} \\
q_{d}
\end{array}\right]=\left[\begin{array}{cc}
\cosh (\gamma d) & -\frac{1}{k \gamma} \sinh (\gamma d) \\
-k \gamma \sinh (\gamma d) & \cosh (\gamma d)
\end{array}\right]\left[\begin{array}{l}
\theta_{0} \\
q_{0}
\end{array}\right]
$$

where $\gamma=\sqrt{\frac{j \omega}{D}}, j=\sqrt{-1}, k$ is the material thermal conductivity, and $d$ is the layer thickness. Recursive application of this transport matrix scheme can thus be used to describe one-dimensional heat flow across several domains. That is,

$$
\left[\begin{array}{l}
\theta_{N} \\
q_{N}
\end{array}\right]=M_{N} M_{N-1} \ldots M_{1}\left[\begin{array}{l}
\theta_{1} \\
q_{1}
\end{array}\right]
$$

where $M_{N}$ is the transport matrix for the specified layer, containing the appropriate layer properties. Given any two of the temperatures or fluxes at either end of a domain, the other two can be found using this method.

In the PEE measurement scheme depicted (Figure 3c), we measured the temperature amplitude (complex value) at the top surface of the stack, $\theta_{H}$, using the 3 -omega method, and the heat flux at the top surface, $q_{H}$, is just the AC power dissipated per unit area. These values are used to walk down the stack and thus determine the temperature amplitude at several positions within the stack. The key insight is to note that any single domain may be divided into an arbitrary number of sub-domains, allowing for the calculation of desired values at any height within the stack. Note, also, that interfaces between layers have been modeled as thin layers $(\sim 1 \mathrm{~nm})$ with low thermal conductivity $\left(1 \mathrm{Wm}^{-1} K^{-1}\right)$ and small heat capacity $\left(10^{5} \mathrm{Jm}^{-3} \mathrm{~K}^{-1}\right)$. 


\section{Electrocaloric effect}

The 1-D heat transport model used for the PEE measurements can be extended to account for a heat source of finite thickness in the middle of a material stack in the case of the ECE measurements. A 1-D model is valid here because the heater (ferroelectric) half width $(b=5 \mu m)$ is much greater than the total thickness of the film stack $(<0.5 \mu \mathrm{m})$ and thus there is minimal lateral spreading within the substrate. Beginning with the transport matrices defined by equation (A.1) for each layer in the stack, we can build up a formalism for the internal heat generation. Considering the stack (Figure 4c), differential heat generated at an infinitesimal layer $d z$ at height $z$, diffuses up toward the surface of the stack and also into the substrate. This allows the stack to be divided into two sub domains: the upper (1) and lower (2) half of the stack, relative to height $z$ within the ferroelectric. From these considerations we have three equations,

$$
\begin{array}{r}
d q_{\text {tot }}=d q_{1}+d q_{2} \\
{\left[\begin{array}{c}
\theta_{t} \\
d q_{t}
\end{array}\right]=\left[\begin{array}{ll}
A_{1} & B_{1} \\
C_{1} & D_{1}
\end{array}\right]\left[\begin{array}{c}
\theta_{z} \\
d q_{z_{1}}
\end{array}\right]} \\
{\left[\begin{array}{c}
\theta_{b} \\
d q_{b}
\end{array}\right]=\left[\begin{array}{ll}
A_{2} & B_{2} \\
C_{2} & D_{2}
\end{array}\right]\left[\begin{array}{c}
\theta_{z} \\
d q_{z_{2}}
\end{array}\right]}
\end{array}
$$

where the subscript $t$ represents the properties of the bottom surface of the sensor, $b$ the properties of the bottom surface of the substrate, $z$ the properties of the ferroelectric layer at the prescribed height of interest, 1) the total transport matrix (i.e., the recursive multiplication of the $M$ matrices for the relevant domains)) for the upper domain, 2 the analogous transport matrix for the lower domain, and $q_{t o t}$ is the total flux generated by the infinitesimal layer.

The fluxes at the top surface (adiabatic) and the bottom surface (semi-infinite) are zero. This allows us to write:

$$
\begin{aligned}
& C_{1} \theta_{z}+D_{1} d q_{z_{1}}=0 \\
& C_{2} \theta_{z}+D_{2} d q_{z_{2}}=0
\end{aligned}
$$

Applying the conservation of flux, equation (A.3), to these equations leads to

$$
\theta_{z}=\left(\frac{-D_{1} D_{2}}{C_{1} D_{2}+C_{2} D_{1}}\right) d q_{t o t}
$$

The volumetric heat generated within the ferroelectric when exposed to an oscillating electric field, $G$, is defined by equation (13). Using equation (14), the temperature response at any plane in the ferroelectric can be calculated by

$$
\theta_{z}=G \int_{0}^{d_{F E}}\left(\frac{-D_{1} D_{2}}{C_{1} D_{2}+C_{2} D_{1}}\right) d z
$$

Equation (A.9) can be applied to the top and bottom surfaces of the ferroelectric layer to determine the average temperature oscillation in the ferroelectric, $\theta_{F E}=$ $\frac{1}{2}\left(\theta_{z=0}+\theta_{z=d_{F E}}\right)$.

We can further extend this matrix formalism to determine an explicit expression for the temperature at the top surface of the stack (i.e. the temperature measured by the sensor). From equation (A.4) and (A.6),

$$
\theta_{t}=A_{1} \theta_{z}+B_{1} d q_{z_{1}}=A_{1} \theta_{z}+B_{1}\left(-\frac{C_{1}}{D_{1}}\right) \theta_{z}
$$

Finally, combining (A.9) and (A.10),

$$
\theta_{t}=G \int_{0}^{d_{F E}}\left(\frac{B_{1} C_{1}-A_{1} D_{1}}{C_{1} D_{2}+C_{2} D_{1}}\right) D_{2} d z
$$

We see in equation (A.11) the complete expression used to relate the surface temperature measured by the heater line $\left(\theta_{\text {Sensor }}\right)$ to $\Sigma$.

[1] M.E. Lines and A.M. Glass, Principles and Applications of Ferroelectrics and Related Materials (OUP Oxford, 1977) p. 129

[2] J. F. Scott, Electrocaloric materials, Annu. Rev. Mater. Res. 41, 229-240 (2011).

[3] R. W. Whatmore, Pyroelectric devices and materials, Rep. Prog. Phys. 49, 1335 (1986).

[4] A. Hadni, Applications of the pyroelectric effect, J. Phys. E 14, 1233 (1981).

[5] A. Cuadras, M. Gasulla, and V. Ferrari, Thermal energy harvesting through pyroelectricity, Sensor Actuat. A-Phys. 158, 132-139 (2010).

[6] Y. Yang, W. Guo, K. C. Pradel, G. Zhu, Y. Zhou, Y. Zhang, Y. Hu, L. Lin, and Z. L. Wang, Pyroelectric nanogenerators for harvesting thermoelectric energy, Nano Lett. 12, 2833-2838 (2012).

[7] A. Kitanovski, U. Plaznik, U. Tomc, and A. Poredo, Present and future caloric refrigeration and heat-pump technologies, Int. J. Refrig. 57, 288-298 (2015).

[8] V. K. Pecharsky, J. Cui, and D. D. Johnson, (magneto)caloric refrigeration: is there light at the end of the tunnel? Phil. Trans. R. Soc. A 374 (2016).

[9] A. G. Chynoweth, Dynamic method for measuring the pyroelectric effect with special reference to barium titanate, 
J. Appl. Phys. 27, 78-84 (1956)

[10] S. B. Lang and F. Steckel, Method for the measurement of the pyroelectric coefficient, dc dielectric constant, and volume resistivity of a polar material, Rev. Sci. Instrum. 36, 929-932 (1965).

[11] L. Pintilie, M. Alexe, I. Pintilie, and I. Boierasu, Thermally stimulated currents in $\mathrm{PbTiO}_{3}$ thin films, Ferroelectrics 201, 217-223 (1997)

[12] H. Okino, Y. Toyoda, M. Shimizu, T. Horiuchi, T. Shiosaki, and K. Matsushige, Thermally stimulated current and polarization fatigue in $\mathrm{Pb}(\mathrm{Zr}, \mathrm{Ti}) \mathrm{O}_{3}$ thin films, Jpn. J. Appl. Phys. 37, 5137 (1998).

[13] I. Lubomirsky and O. Stafsudd, Invited review article: Practical guide for pyroelectric measurements, Rev. Sci. Instrum. 83, 051101 (2012)

[14] L. E. Garn and E. J. Sharp, Use of lowfrequency sinusoidal temperature waves to separate pyroelectric currents from nonpyroelectric currents. Part I. Theory, J. Appl. Phys 53, 8974-8979 (1982)

[15] E. J. Sharp and L. E. Garn, Use of lowfrequency sinusoidal temperature waves to separate pyroelectric currents from nonpyroelectric currents. Part II. Experiment, J. Appl. Phys. 53, 8980-8987 (1982).

[16] B. Bhatia, J. Karthik, T. Tong, D. G. Cahill, L. W. Martin, and W. P. King, Pyroelectric current measurements on $\mathrm{PbZr}_{0.2} \mathrm{Ti}_{0.8} \mathrm{O}_{3}$, J. Appl. Phys. 112, 104106 (2012)

[17] T. Tong, J. Karthik, L. W. Martin, and D. G. Cahill, Secondary effects in wide frequency range measurements of the pyroelectric coefficient of $\mathrm{Ba}_{0.6} \mathrm{Sr}_{0.4} \mathrm{TiO}_{3}$, Phys. Rev. B 90, 155423 (2014).

[18] S. Kar-Narayan, S. Crossley, X. Moya, V. Kovacova, J. Abergel, A. Bontempi, N. Baier, E. Defay, and N. D. Mathur, Direct electrocaloric measurements of a multilayer capacitor using scanning thermal microscopy and infra-red imaging, Appl. Phys. Lett. 102, 032903 (2013)

[19] F. L. Goupil, A. Berenov, A. K Axelsson, M. Valant, and N. M. Alford, Direct and indirect electrocaloric measurements on $<001>-\mathrm{PbMg}_{1 / 3} \mathrm{Nb}_{2 / 3} \mathrm{O}_{3}-30 \mathrm{PbTiO}_{3}$ single crystals, J. Appl. Phys. 111, 124109 (2012)

[20] X. Li, X. S Qian, H. Gu, X. Chen, S. G. Lu, M. Lin, F. Bateman, and Q. M. Zhang, Giant electrocaloric effect in ferroelectric poly(vinylidenefluoride-trifluoroethylene) copolymers near a first-order ferroelectric transition, Appl. Phys. Lett. 101, 132903 (2012)

[21] L. W. Martin, Y. H Chu, and R. Ramesh, Advances in the growth and characterization of magnetic, ferroelectric, and multiferroic oxide thin films, Mater. Sci. Eng. R-Rep. 68, 89-133 (2010).

[22] D. G. Schlom, L. Q Chen, C. B Eom, K. M. Rabe, S. K. Streiffer, and J. M Triscone, Strain tuning of ferroelectric thin films, Annu. Rev. Mater. Res. 37, 589-626 (2007).

[23] G. Chen, J. Zhao, S. Li, and L. Zhong, Origin of thickness dependent dc electrical breakdown in dielectrics, Appl. Phys. Lett. 100, 222904 (2012)

[24] A. S. Mischenko, Q. Zhang, J. F. Scott, R. W. Whatmore, and N. D. Mathur, Giant electrocaloric effect in thin-film $\mathrm{PbZr}_{0.95} \mathrm{Ti}_{0.05} \mathrm{O}_{3}$, Science 311, 1270-1271 (2006).

[25] A. S. Mischenko, Q. Zhang, R. W. Whatmore, J. F. Scott, and N. D. Mathur, Giant electrocaloric effect in the thin film relaxor ferroelectric $0.9 \mathrm{PbMg}_{13} \mathrm{Nb}_{23} \mathrm{O}_{3} 0.1 \mathrm{PbTiO}_{3}$ near room temperature, Appl. Phys. Lett. 89, 242912 (2006).

[26] T. M. Correia, J. S. Young, R. W. Whatmore, J. F. Scott, N. D. Mathur, and Q. Zhang, Investigation of the electrocaloric effect in a $\mathrm{PbMg}_{2 / 3} \mathrm{Nb}_{1 / 3} \mathrm{O}_{3}-\mathrm{PbTiO}_{3}$ relaxor thin film, Appl. Phys. Lett. 95, 182904 (2009).

[27] H. Chen, T. L Ren, X. M Wu, Y. Yang, and L. T Liu, Giant electrocaloric effect in lead-free thin film of strontium bismuth tantalite, Appl. Phys. Lett. 94, 182902 (2009)

[28] Y. Liu, J. F. Scott, and B. Dkhil, Direct and indirect measurements on electrocaloric effect: Recent developments and perspectives, Appl. Phys. Rev. 3, 031102 (2016)

[29] Xinyu Li, Sheng-Guo Lu, Xiang-Zhong Chen, Haiming Gu, Xiao-Shi Qian, and Q. M. Zhang, Pyroelectric and electrocaloric materials, J. Mater. Chem. C 1, 23-37 (2013).

[30] B. Neese, B. Chu, S. G Lu, Y. Wang, E. Furman, and Q. M. Zhang, Large electrocaloric effect in ferroelectric polymers near room temperature, Science 321, 821-823 (2008)

[31] B. Peng, H. Fan, and Q. Zhang, A giant electrocaloric effect in nanoscale antiferroelectric and ferroelectric phases coexisting in a relaxor $\mathrm{Pb}_{0.8} \mathrm{Ba}_{0.2} \mathrm{ZrO}_{3}$ thin film at room temperature, Adv. Funct. Mater. 23, 2987-2992 (2013)

[32] T. Tong, J. Karthik, R. V. K. Mangalam, L. W. Martin, and D. G. Cahill, Reduction of the electrocaloric entropy change of ferroelectric $\mathrm{PbZr}_{1-x} \mathrm{Ti}_{x} \mathrm{O}_{3}$ epitaxial layers due to an elastocaloric effect, Phys. Rev. B 90, 094116 (2014).

[33] D. G. Cahill, M. Katiyar, and J. R. Abelson, Thermal conductivity of a-Si:H thin films, Phys. Rev. B 50, 6077-6081 (1994).

[34] S. Pandya, A. R. Damodaran, R. Xu, S. L Hsu, J. C. Agar, and L. W. Martin, Strain-induced growth instability and nanoscale surface patterning in perovskite thin films, Sci. Rep. 6, 26075 (2016).

[35] J. Karthik, A. R. Damodaran, and L. W. Martin, Epitaxial ferroelectric heterostructures fabricated by selective area epitaxy of $\mathrm{SrRuO}_{3}$ using an $\mathrm{MgO}$ mask, Adv. Mater. 24, 1610-1615 (2012).

[36] L. Pintilie, I. Vrejoiu, D. Hesse, and M. Alexe, The influence of the top-contact metal on the ferroelectric properties of epitaxial ferroelectric $\mathrm{Pb}\left(\mathrm{Zr}_{0.2} \mathrm{Ti}_{0.8}\right) \mathrm{O}_{3}$ thin films, J. Appl. Phys. 104, 114101 (2008)

[37] J. Karthik, A. R. Damodaran, and L. W. Martin, Effect of $90^{\circ}$ domain walls on the low-field permittivity of $\mathrm{PbZr}_{0.2} \mathrm{Ti}_{0.8} \mathrm{O}_{3}$ thin films, Phys. Rev. Lett. 108, 167601 (2012).

[38] C. Dames, Measuring the thermal conductivity of thin films: 3 omega and related electrothermal methods, Annu. Rev. Heat Transfer (Begell House, New York, 2013) Vol. 16 p. 7

[39] Jaeger J. C. and Carslaw, H. S., Conduction of heat in solids, (Clarendon Press, Oxford, 1959) p. 109, 2nd ed

[40] L. B. Schein, P. J. Cressman, and L. E. Cross, Electrostatic measurements of tertiary pyroelectricity in partially clamped $\mathrm{LiNbO}_{3}$, Ferroelectrics 22, 945-948 (1978). 


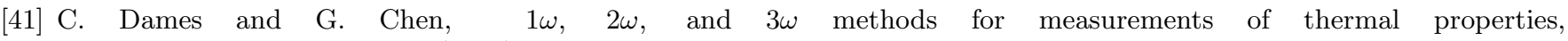
Rev. Sci. Instrum. 76, 124902 (2005).

[42] L. Lu, W. Yi, and D. L. Zhang, $3 \omega$ method for specific heat and thermal conductivity measurements, Rev. Sci. Instrum. 72, 2996-3003 (2001)

[43] D. G. Cahill, M. Katiyar, and J. R. Abelson, Thermal conductivity of a-Si:H thin films, Phys. Rev. B 50, 6077-6081 (1994).

[44] D. G. Cahill, Erratum: thermal conductivity measurement from 30 to $750 \mathrm{~K}$ : The $3 \omega$ method [rev. sci. instrum. 61,802 (1990)], Rev. Sci. Instrum. 73, 3701-3701 (2002).

[45] J. D. Zook and S. T. Liu, Pyroelectric effects in thin film, J. Appl. Phys. 49, 4604-4606 (1978)

[46] B. C. Gundrum, D. G. Cahill, and R. S. Averback, Thermal conductance of metal-metal interfaces, Phys. Rev. B 72, 245426 (2005) 